\title{
Observações hidrológicas e resultados de modelagem no espalhamento sazonal e espacial da pluma de água Amazônica
}

\begin{abstract}
Alex Costa da SILVA, Maria de Lourdes Souza SANTOS², Moacyr Cunha ARAUJO³, Bernard BOURLÈS ${ }^{4}$
\section{RESUMO}

Neste estudo são apresentadas as análises espaciais e temporais dos dados sinóticos coletados durante quatro campanhas oceanográficas do programa "REVIZEE/SCORE-N" realizadas em março-abril de 1995, outubro-novembro de 1997, abrilmaio de 1999 e agosto-setembro de 2001. Em complemento às análises dos dados hidrológicos, foram utilizados resultados do modelo numérico CLIPPER - Experimento ATL6 (1/6 deg.). A ênfase deste artigo é avaliar o deslocamento sazonal e espacial da pluma de água Amazônica nas regiôes localizadas à sudoeste, noroeste e em frente à foz do rio Amazonas. Dados hidrológicos e resultados do modelo demonstram que durante os meses de março-maio a pluma do rio Amazonas move-se para sudoeste, ao longo da costa norte brasileiro, tendendo a diminuir o deslocamento da pluma para sudoeste durante os meses de agosto-setembro. De outubro a novembro, não foi observado ou registrado o deslocamento da pluma para sudoeste. Resultados do modelo revelam anéis gerados pela retroflexão da Corrente Norte do Brasil (CNB) em torno de $7^{\circ} \mathrm{N}$, e também a influência de água proveniente do rio Amazonas em volta desses anéis durante o mês de agosto.
\end{abstract}

PaLAVRaS-CHAVE: Pluma do rio Amazonas; anéis; Corrente Norte do Brasil; modelo Atlântico; dados do REVIZEE.

\section{Hydrological observations and modeling results of the seasonal and spatial spread of the Amazonian water plume}

\section{ABSTRACT}

In this study, we present surface synoptic data collected during four oceanographic cruises undertaken in March-April 1995, October-November 1997, April-May 1999 and August-September 2001 in the Brazilian Program "REVIZEE/SCORE-NO" and a combination of model results (1/6 deg.). The emphasis of this paper is to point out the monthly changes on the spreading of the Amazon plume over the external Amazon shelf area and the eastern region of the Amazon River. Hydrographic data and model results demonstrated that during March-May, the Amazon plume moved southwestward along the northern coast of Brazil, decreasing during August-September; but this was not observed during $O_{\text {ctober-November. Model results revealed }}$ ring signatures shedding from the North Brazil Current (NBC). Results also showed that during August the Amazon plume encircling the NBC retroflection was around $7^{\circ} \mathrm{N}$.

KEY WORDS: Amazon plume; rings; North Brazil Current; Atlantic model; REVIZEE data

\footnotetext{
${ }^{1}$ Instituto de Ciências do Mar

${ }^{2}$ Universidade Federal do Pará

${ }^{3}$ Universidade Federal de Pernambuco

${ }^{4}$ Institut de Rechercher pour le Développement
} 


\section{INTRODUÇÃO}

A borda oeste do oceano Atlântico equatorial é considerada, nos últimos anos pela comunidade científica, como uma zona de grande importância para compreensão da circulação oceânica global o que a transforma numa região-chave para o melhor entendimento dos mecanismos de transferência de calor e massa que ocorrem entre os hemisférios.

A região é influenciada por forçantes de natureza distintas como, por exemplo, as correntes de fronteira oeste, ventos alísios, e pelo aporte de água doce oriunda do rio Amazonas. Este último influi na distribuição de salinidade nas águas tropicais e, de forma indireta, na variabilidade das propriedades termodinâmicas em larga extensão sobre o oceano Atlântico tropical. Influencia também no estoque de nutrientes na plataforma continental, porém algumas evidências quantitativas de águas amazônicas reconhecem o relativo suprimento de nutrientes a partir do rio e também de fontes advectivas (Gibbs, 1972; Milliman e Boyle, 1975; Sprintall e Tomczac, 1992; Pailler et al., 1999; Silva et al., 2005a,b; Silva et al., 2007a,b; Ffield, 2007; Subramaniam et al., 2008). Calef e Grice (1967) mostram que as lentes de águas amazônicas afetam a diversidade e a quantidade de certas comunidades planctônicas ao largo da foz do rio Amazonas.

No sentido de estudar a influência das águas provenientes do rio Amazonas na costa norte brasileira, foi selecionada a área que compreende a região da plataforma continental do Amazonas (PCA) e a região oceânica adjacente (Figura 1), que fazem parte da Zona Econômica Exclusiva Norte Brasileira (ZEE-NORTE). Nessa região foram realizadas as campanhas oceanográficas do programa REVIZEE/SCORE-NORTE (Programa Nacional de Avaliação do Potencial Sustentável de Recursos Vivos da Zona Econômica Exclusiva/Subcomitê da Região Norte). Este programa obteve muitas informaçōes acerca da variabilidade espaço-sazonal das propriedades termohalinas ao longo da fronteira oeste brasileira do oceano Attântico tropical. O estudo dessas informaçôes possibilitou aprofundar o conhecimento da variabilidade espacial e sazonal da pluma de água amazônica, associada à dinâmica oceânica de meso-escala na plataforma continental Norte do Brasil e área oceânica adjacente. Expedições e experimentos anteriores, ou se restringiram exclusivamente à região da plataforma continental Amazônica (limitada, portanto, à isóbata de $100 \mathrm{~m}$ ), como por exemplo, o Projeto AMASSEDS, ou se dedicaram à realização de perfis oceanográficos (transeções) em longitudes específicas e/ou limitados à região oceânica (profundidades superiores a $500 \mathrm{~m}$ ) (ex: Schott et al., 1998; Bourlès et al., 1999a,b).

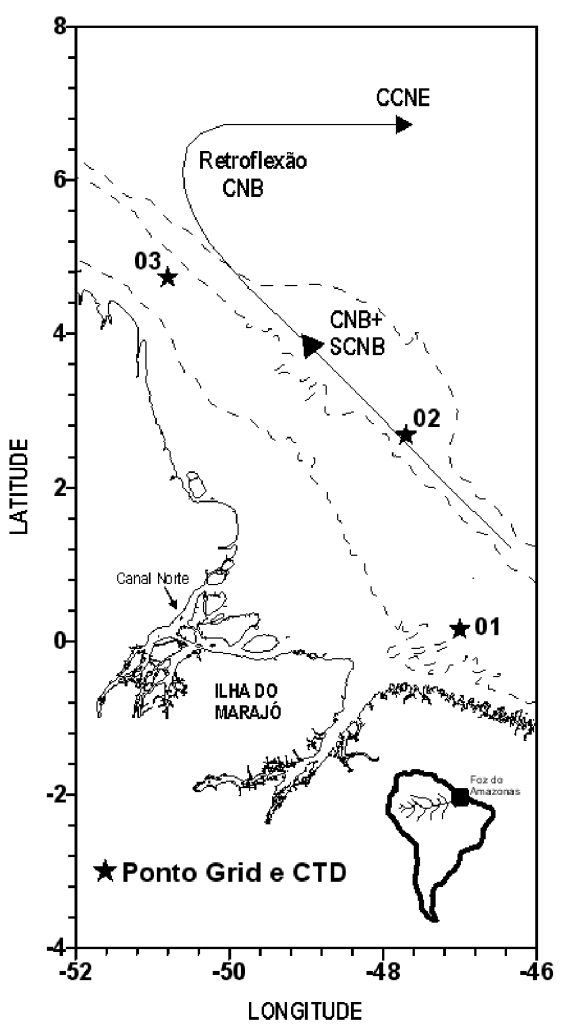

Figura 1 - Mapa da plataforma continental do Amazonas (PCA) e área oceânica adjacente, demonstrando a localização das estações hidrológicas durante as campanhas oceanográficas REVIZEE/SCORE-NORTE (estrelas no mapa), observando-se também a localização dos três pontos de grides obtidos do modelo ATL6. As setas contínuas mostram a influência da Corrente Norte do Brasil (CNB)/Subcorrente Norte do Brasil (SCNB), a Contracorrente Norte Equatorial (CCNE) e a sua retroflexão na região oceânica. As linhas pontilhadas representam as isóbatas de 20, 100 e $2000 \mathrm{~m}$.

\section{DINÂMICA NA PLATAFORMA CONTINENTAL DO AMAZONAS E ÁREA OCEÂNICA ADJACENTE}

\section{A) DESCARGA DO RIO AMAZONAS}

A bacia hidrográfica do rio Amazonas se estende desde os Andes, cruza a floresta Amazônica até o Atlântico, e cobre uma área de aproximadamente $7 \times 10^{6} \mathrm{~km}^{2}$. É considerada a maior do mundo (Nittrouer e DeMaster, 1986), descarrega em torno de $180.000 \mathrm{~m}^{3} \cdot \mathrm{s}^{-1}$ ou $18 \mathrm{~Sv}$ de água doce no oceano Atlântico e contribui com cerca de $18 \%$ do total de água doce continental lançada nos oceanos (Oltman, 1968; Müller-Karger $e t$ al., 1988). Essa vazão varia sazonalmente, com máximo em torno de 0,24 Sv em maio, e mínimo em torno de 0,08 Sv em novembro (Figura 2). O maior volume de água doce (cerca de $65 \%$ da descarga total) passa por meio do canal Norte, que durante o período de máxima descarga veicula uma vazão de cerca de $160.000 \mathrm{~m}^{3} \cdot \mathrm{s}^{-1}$ o que 
corresponde a uma velocidade da ordem de $80 \mathrm{~cm} \mathrm{~s}^{-1}$ (Geyer et al., 1996). A água doce proveniente do rio Amazonas se mistura com águas oceânicas formando uma pluma superficial de baixa salinidade - pluma de água amazônica $(S<34)$ que é transportada para noroeste pela corrente Norte do Brasil CNB (Silva et al., 2007b).

\section{B) CORRENTE NORTE DO BRASIL (CNB)}

$\mathrm{Na}$ região oceânica adjacente à plataforma continental a circulação próxima da superfície é caracterizada pela presença da corrente Norte do Brasil e sua componente em subsuperfície a subcorrente Norte do Brasil (SCNB). Estas são formadas a partir da bifurcação da corrente Sul Equatorial, que alimenta o sistema $\mathrm{CNB} / \mathrm{SCNB}$. Após essa bifurcação, o sistema de CNB/SCNB transporta água quente do Atlântico sul para noroeste, ao longo da costa brasileira, cruzando a linha do equador em direção ao Atlântico Norte. A CNB varia de direção ao longo da costa norte brasileira, de acordo sobretudo com os padróes dos ventos e da localização no trajeto da corrente.

Ao longo de $44^{\circ} \mathrm{W}$ o sistema da CNB/SCNB transporta água em torno de $35 \mathrm{~Sv}$ a $36 \mathrm{~Sv}\left(1 \mathrm{~Sv}=10^{6} \mathrm{~m}^{3} \mathrm{~s}^{-1}\right)$ em direção ao Equador, com uma amplitude de variação sazonal média de 3 Sv (Johns et al., 1998; Schott et al., 1998; Bourlès et al., 1999a). Em geral, a máxima velocidade da CNB é localizada na isopicna de 24,5 , com fluxo em torno de 60-100 cm.s. . Segundo Richardson et al. (1994), a CNB flui ao longo da quebra da PCA sobre o talude continental, predominantemente no sentido noroeste, e com velocidades da ordem de 50-100 cm.s. ${ }^{-1}$. Johns et al. (1998) estimaram valores de transporte da $\mathrm{CNB}$ adjacente à costa Amazônica $\left(4^{\circ} \mathrm{N}-45^{\circ} \mathrm{W}\right)$ entre $35 \mathrm{~Sv}$ nos meses de julho-agosto e $13 \mathrm{~Sv}$ em abril-maio, com uma média anual de aproximadamente 26 Sv (Figura 2).

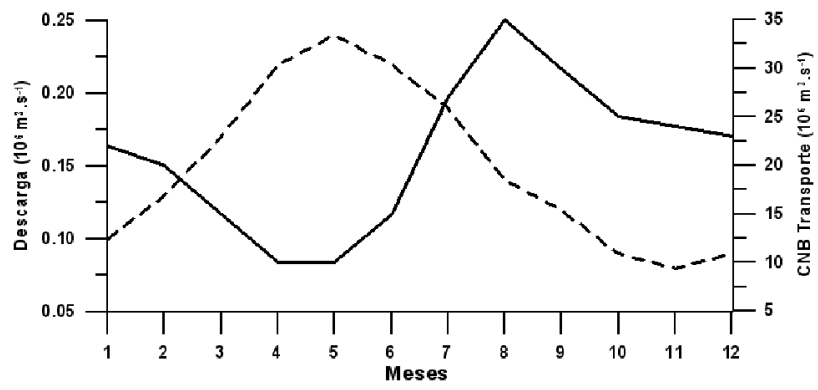

Figura 2 - Variação sazonal da descarga do rio Amazonas e da intensidade da Corrente Norte do Brasil (CNB), adaptado de Johns et al. (1998) e de Geyer et al. (1996).

\section{C) AÇÃO EÓLICA}

Além da forte influência das correntes de fronteira, a costa norte brasileira tem influência dos ventos alísios que atingem sua máxima velocidade entre dezembro e maio, período que corresponde aos alísios de nordeste que atuam quase perpendicularmente à costa e podem atingir velocidades medias de $18 \mathrm{~m} . \mathrm{s}^{-1}$ em fevereiro. De junho à novembro estes ventos mudam de posição, sopram de sudeste e atingem sua menor intensidade, abaixo de $12 \mathrm{~m} . \mathrm{s}^{-1}$ (Nittrouer e DeMaster, 1996). Dados de médias climatológicas mensais de ventos fornecidos pelo National Centers for Environmental Prediction (NCEP) para o período de 1997 a 2002 (Silva et al., 2007a), mostram a predominância de ventos alísios de nordeste em maio, período este em que a Zona de Convergência Intertropical (ZCIT) se encontra mais próxima do Equador e há mudança na variação de direção dos ventos alísios de nordeste para sudeste em junho (Figura 3).

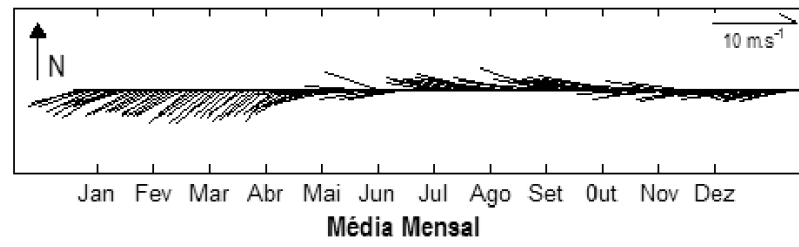

Figura 3 - Média mensal da direção do vento na região de estudo, mostra os ventos alísios de nordeste no mês de maio e a mudança na direção dos ventos alísios para sudeste no mês de junho, adaptado de Silva et al. (2007a).

\section{MATERIAIS E MÉTODOS}

\section{DADOS COLETADOS NO MAR}

Os dados foram coletados durante quatro campanhas oceanográficas realizadas pelo Navio Oceanográfico Antares na região da Plataforma Continental do Amazonas (PCA) e região oceânica adjacente (Figura 1). Essas campanhas foram efetivadas dentro do contexto do Programa REVIZEE.

As quatro campanhas foram realizadas em épocas diferentes do ano, associadas às distintas situações de aporte continental de água doce, ou seja: outono boreal - período de descarga mínima do rio Amazonas (outubro-novembro de 1997), final de inverno e primavera boreal - descarga máxima do rio Amazonas (março-abril de 1995/abril-maio de 1999), e verão boreal - período de transição de máxima para mínima descarga (julho-setembro de 2001). No sentido de estudar a influência das águas provenientes do rio Amazonas na costa norte brasileira, foram analisados os parâmetros físicoquímicos de salinidade, silicato e fosfato, considerando que a contribuição fluvial é a principal responsável pelo aporte de nutrientes e na diminuição de salinidade nas águas superficiais das regiōes costeiras.

\section{A) SALINIDADE}

O conjunto dos dados analisados corresponde a análise previamente escolhida de 12 perfis verticais contínuos 
de temperatura e salinidade obtidos com equipamento Conductivity, Temperature e Depth - CTD da Sea Bird Electronics SBE911plus, equipado com bomba centrifuga e sensores de alta resolução para medições de condutividade (resolução $\left.=0,00004 \mathrm{~S} \mathrm{~m}^{-1}\right)$, temperatura $\left(\right.$ resolução $=0,0003^{\circ}$ C) e pressão (resolução $=0,068 \mathrm{db}$ ). A salinidade foi calculada com os algoritmos da escala prática (PSS-178) adotada pela UNESCO, em função dos valores de condutividade elétrica, temperatura e pressão (UNESCO, 1981).

Para estimar a influência da pluma d'água Amazônica (<34 ups) na plataforma continental Norte do Brasil e área oceânica adjacente, foram analisados os registros das médias mensais de salinidade do modelo e dos dados coletados in situ, em três pontos da malha de amostragem, localizados aproximadamente a sudeste da foz do rio Amazonas - Ponto $01\left(47,0^{\circ} \mathrm{W}-0,15^{\circ} \mathrm{N}\right)$, a $300 \mathrm{~km}$ perpendicularmente da foz do rio Amazonas - Ponto $2\left(47,7^{\circ} \mathrm{W}-2,68^{\circ} \mathrm{N}\right)$ e um gride localizado a noroeste da foz do rio Amazonas - Ponto 03 $\left(50,8^{\circ} \mathrm{W}-4,73^{\circ} \mathrm{N}\right)$ nas quatro distintas épocas de campanhas oceanográficas do Programa REVIZEE (estrelas no mapa da Figura 1).

\section{B) SILICATO E FOSFATO}

Para determinação do silicato e fosfato as amostras de água foram filtradas em membranas de $0,45 \mu \mathrm{m}$, armazenadas em frascos de polietileno de $500 \mathrm{~mL}$ e congeladas. Em laboratório, as amostras de água foram descongeladas e as análises feitas em duplicatas para determinação do fosfato e silicato, aplicandose a metodologia descrita em Grasshoff et al. (1983). A água utilizada para determinação era proveniente de um ALPHA Q, e todas as leituras dos nutrientes foram efetivadas através de espectrofotômetro.

A concentração do fosfato $\left(\mathrm{PO}_{4}^{3-}\right)$ foi obtida com a utilização do heptamolibdato de amônio ( $(\mathrm{NH}) \mathrm{Mo} \mathrm{O} .4 \mathrm{H} \mathrm{O}) \mathrm{em}$ meio ácido $\left(\mathrm{H}^{+}\right)$, formando o complexo fosfomolibdato. Este complexo é então reduzido pelo ácido ascórbico $\left(\mathrm{C}_{6} \mathrm{H}_{8} \mathrm{O}_{6}\right)$, numa reação catalisada pelo antimônio tartarato de potássio $\left(\mathrm{K}(\mathrm{SbO}) \mathrm{C}_{4} \mathrm{H}_{4} \mathrm{O}_{6}\right)$, formando um complexo $\left(\left(\mathrm{NH}_{4}\right)_{3} \mathrm{PO}_{4} \mathrm{Mo}_{12} \mathrm{O}_{36}\right)$ de coloração azul que tem absorção máxima em $880 \mathrm{~nm}$.

A análise para silicato também foi feita com o heptamolibdato de amônio em meio ácido, porém, com a formação do ácido silicomolibdíco ( $\mathrm{H}$ SiMo O ), e leitura em $810 \mathrm{~nm}$. Para evitar à interferência do fosfato presente na amostra foi empregado ácido oxálico $\left((\mathrm{COOH}) \cdot 2 \mathrm{H}_{2} \mathrm{O}\right)$.

\section{DADOS DO MODELO HIDRODINÂMICO (O PROJETO CLIPPER -} EXPERIMENTO ATL6)

O projeto CLIPPER foi desenvolvido com base na realização de quatro configuraçôes básicas (ou experimentos) simuladas com a versão 8.1 do código numérico paralelizado
OPA, desenvolvido pelo LODYC (Paris). Os resultados apresentados e utilizados neste trabalho foram gerados a partir do Experimento ATL6, composto por uma malha de gride horizontal médio de 1/60 (Gride Mercator), utilizada por diferentes pesquisadores como a simulação de referência para o oceano Atlântico tropical (Madec et al., 1998; Michel e Treguier, 2002; Arhan et al., 2006; Treguier et al., 2006; Gulev et al., 2007).

A batimetria utilizadas no Experimento ATL6 foi calculada de Smith e Sandwell (1997) e as condiçôes iniciais de temperatura $(\mathrm{T})$ e salinidade $(\mathrm{S})$ foram obtidas a partir da climatologia de Reynaud et al. (1998), derivada das mediçôes realizadas nas campanhas do Programa WOCE. Nas simulações foram utilizadas forçantes "realistas" de ventos, fluxos de calor e de água doce, obtidas para o período do Programa WOCE (1980-1999). Estas informações foram complementadas com os dados diários do Centre Européen de Prévision Météorologique à Moyen Terme (ECMWF), para os demais períodos de simulação. Ao mesmo tempo, uma nova climatologia mensal de aporte fluvial de água doce foi fornecida pela UNESCO (1996).

Os resultados do modelo para o Experimento ATL6 representam uma média de cinco dias, utilizado no $1^{\circ}$ nível sigma do modelo ( $\approx 6 \mathrm{~m}$ de profundidade). Em um ano de simulação foram requeridas 5000 horas de processamento computacional. Maiores informações podem ser obtidas na documentação específica do Projeto CLIPPER (Barnier et al., 2001) ou em (http://www.ifremer.fr/lpo/clipper/), acesso: 16/10/08.

Para o desenvolvimento deste trabalho os campos numéricos de velocidade e salinidade foram obtidos na mesma época dos quatro cruzeiros do Programa REVIZEE, a fim de associar a circulação de grande escala às observaçôes de mesoescala disponíveis em uma área relativamente reduzida. O funcionamento do modelo foi aplicado particularmente aos estudos do Atlântico equatorial, e a parametrização de mistura foi escolhida após uma série de testes, conforme as simulaçōes e validaçôes para reproduzir as correntes de fronteiras do Atlântico equatorial (Michel e Treguier, 2002). Arhan et al. (2006) usaram a mesma simulação para reprodução do ciclo anual da subcorrente Equatorial.

\section{RESULTADOS E DISCUSSÃO}

Com base em medidas in situ e de resultados do Experimento numérico ATL6 do projeto CLIPPER, foi possível avaliar o deslocamento sazonal e espacial da pluma de água amazônica, durante os períodos de campanhas oceanográficas do Programa REVIZEE/SCORE-N, associando-a com a variabilidade sazonal da dinâmica oceânica local (ventos, correntes, etc.). 
- OBSERVACÕES IN SITU E REGISTROS NUMÉRICOS DAS MÉDIAS MENSAIS DE SALINIDADE

O gráfico gerado a partir dos resultados de médias mensais nos anos de 1995, 1997, 1999 e 2001 para a localidade de $47^{\circ} \mathrm{W}-0.15^{\circ} \mathrm{N}$ (a sudeste da foz do rio Amazonas - Estação 1, Figura 1), registrou águas com baixo valor de salinidade ( $<35$ ups) entre fevereiro e maio, tendendo a aumentar sua salinidade nos meses subseqüentes. Os resultados do registro numérico do modelo também demonstram pequena diminuição nos valores de salinidade entre fevereiro e maio, com tendência a aumentar nos meses subseqüentes, de junho a dezembro (Figura 4a). índices de precipitação na região (Evans et al., 2000), o qual ocasionou baixa influência da descarga do rio Amazonas na região oceânica durante este período.

Análises das estações in situ localizadas a noroeste da foz do rio Amazonas $\left(50.8^{\circ} \mathrm{W}\right.$ e $4.73^{\circ} \mathrm{N}$ - Estação 03) indicam a presença de águas amazônicas, principalmente durante a campanha oceanográfica realizada em setembro/2001 (salinidade $<25$ ups) (Figura 4c). Estes baixos valores de salinidade observados em setembro neste local, são devido ao processo de aprisionamento de água Amazônica associados a retroflexão da $\mathrm{CNB}\left(7^{\circ} \mathrm{N}-50,5^{\circ} \mathrm{W}\right)$ e ao tempo de deslocamento que as águas amazônicas provindas da foz do
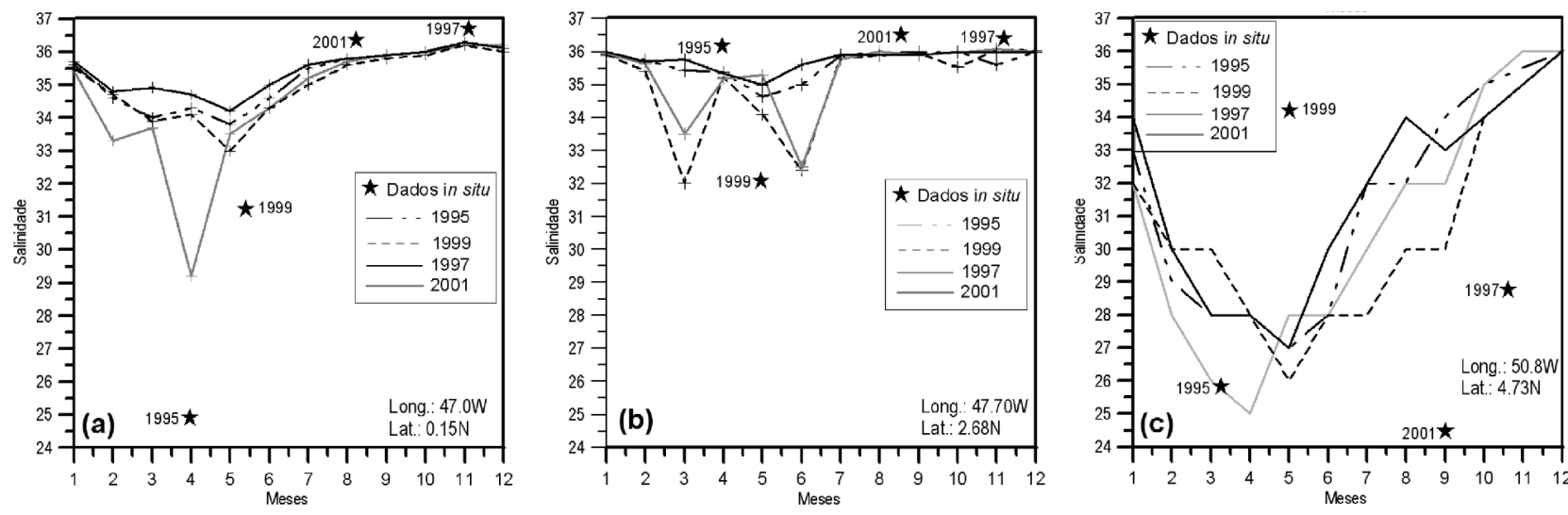

Figura 4 - Média mensal de salinidade obtida por meio de coletas hidrográficas e do experimento numérico (ATL6), em três pontos (Figura 1) localizados, 1) a sudeste da foz do rio Amazonas (47, $0^{\circ} \mathrm{W}-0,15^{\circ} \mathrm{N}$ - Estação 01, Figura 4a), 2) a aproximadamente a $300 \mathrm{~km}$ perpendicularmente da foz do rio Amazonas $\left(47,7^{\circ} \mathrm{W}-2,68^{\circ} \mathrm{N}\right.$ - Estação 02, Figura 4b) e um 3) ponto localizado a noroeste da foz do rio Amazonas $\left(50,8^{\circ} \mathrm{W}-4,73^{\circ} \mathrm{N}\right.$ - Estação 3, Figura 4c).

Lentz (1995a,b) também discutem sobre a pluma Amazônica atuante na plataforma continental do Amazonas, acordando os resultados com a variabilidade da descarga do rio e a atuação do vento local. Neste trabalho também foi possível mostrar que a dispersão da pluma próxima da costa está diretamente relacionada com a intensidade/direção dos ventos alísios. Nos meses de março a maio, período este em que a ZCIT se encontra mais próxima do Equador, os alísios de nordeste bloqueiam a pluma próxima da costa, ocorrendo a sua dispersão para sudeste, atingindo a plataforma continental do Pará (Figura 4a)

As médias mensais do modelo registram influência da pluma de água Amazônica que atinge em torno de $300 \mathrm{~km}$ de distância perpendicular à foz do rio Amazonas (Estação 02) entre março e junho de 1995, 1999 e 2001 . Análises dos dados in situ, indicam a influência da água amazônica em maio/1999 (Figura 4b).

O experimento numérico ATL6 realizado em 1997 nos dois grides - Estação 01 e 02 (Figura 4a e 4b) não apresentou registro de influência de água Amazônica. Isso pode ser conseqüência do evento do El-Niño forte, associado a baixos rio Amazonas atingem esta região (o período de máxima vazão do rio Amazonas no oceano Atlântico ocorre em maio). Os resultados das médias mensais de salinidade simuladas para esta região estão claramente relacionados aos períodos de máxima e mínima vazão do rio Amazonas, registrando os menores valores de salinidade (<29 ups) de março a maio, correspondendo aos valores observados na campanha oceanográfica de março/2005 e os maiores valores de salinidade (> 35 ups) nos meses de novembro e dezembro (Figura 4c).

\section{NUTRIENTES NA PLUMA AMAZÔNICA}

Durante a campanha oceanográfica de abril-maio/1999 foram observados os maiores valores de silicato na estação localizada a sudoeste da foz do rio Amazonas (Estação 01 - 2,72 $\mu$ mol.L $\left.L^{-1}\right)$ e na estação da região oceânica situada em frente da foz do rio Amazonas (Estação 02 - 2,47 $\mu \mathrm{mol} . \mathrm{L}^{-1}$ ) (Figura 5a). $\mathrm{Na}$ campanhas oceanográficas de outubro-novembro/1997 e agosto-setembro/2001, os valores silicatos foram menores nas estaçōes $01\left(1,81\right.$ - 0,85 $\mu$ mol.L $\left.\mathrm{L}^{-1}\right)$ e $02\left(0,91\right.$ - 0,60 $\left.\mu \mathrm{mol} . \mathrm{L}^{-1}\right)$ em relação aos valores observados nesses locais em maio/1999 (Figura 5a). Os altos valores estão coincidentes com o período de máxima descarga do rio Amazonas (Fig 4a, 4b e 5a). Na 
estação localizada a noroeste da foz do rio Amazonas (Estação 03), Santos (2004) detectou um máximo valor de clorofila $a$ $\left(41,45 \mathrm{mg} \cdot \mathrm{m}^{-3}\right)$ em setembro/2001. Nessa mesma localidade e período foi observado o máximo valor de silicato $(41,1$ $\mu$ mol. $\left.L^{-1}\right)$ e fosfato $\left(0,27 \mu\right.$ mol. $\left.L^{-1}\right)$ (Figura 5 a e $\left.5 b\right)$. No entanto, nas campanhas realizadas em novembro/1997 e maio/1999, os valores para silicato e fosfato também foram elevados na Estação 03, o que demonstra a continua presença das águas oriundas do rio Amazonas em direção noroeste da sua foz (Figura 5a e 5b).

\section{INFLUÊNCIA DA PLUMA AMAZÔNICA EM VOLTA DOS ANÉIS OU RINGS FORMADOS PELA RETROFLEXÃO DA CNB}

Conforme as observaçóes coletadas in situ e registros históricos (Oltman, 1968; Lentz e Limeburner, 1995; Silva, et al., 2005a; Silva et al., 2007b), a descrição sazonal do modelo

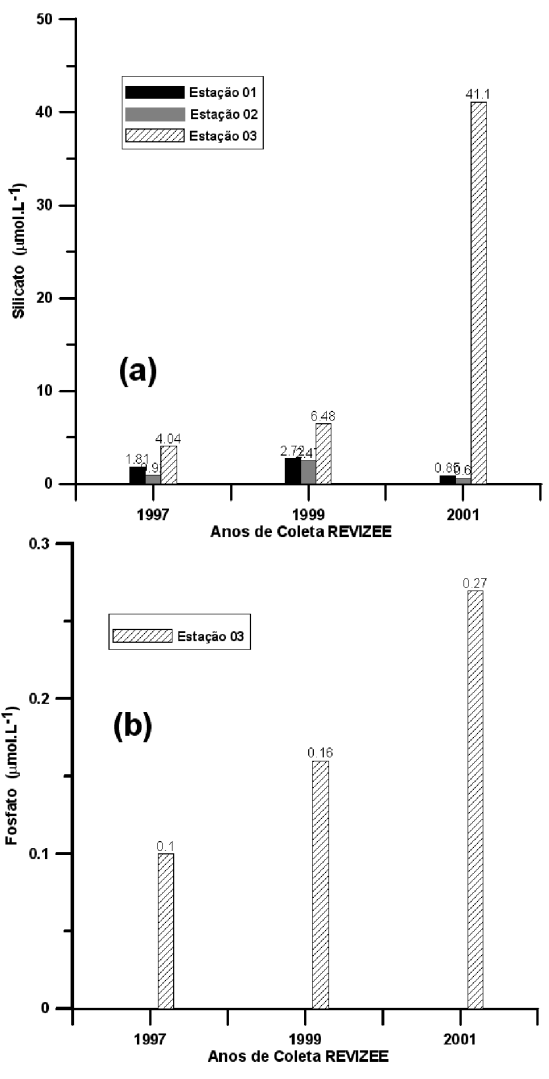

Figura $\mathbf{5}$ - a) Variação espacial e temporal dos valores de silicato nas três estações de coletas hidrológicas (Estações 01, 02 e 03); b) Variação temporal do fosfato na estação localizada a noroeste da foz do rio Amazonas (Estação 03).

relata bem a variabilidade da descarga do rio Amazonas, tendo registrado sua máxima descarga nos meses de abril/ maio, decréscimo em agosto/setembro e mínima descarga em novembro (Figura 6). Destaca-se também que os resultados do modelo indicaram que a distribuição da pluma de água de baixo valor de salinidade está diretamente relacionada à variabilidade sazonal e espacial das correntes de fronteiras atuantes na região de estudo (Figura 2 e Figura 6).

Hu et al. (2004) ao associar imagens da cor do oceano SeaWIFS (no período de setembro 1997 - junho 2002) com o objetivo de evidenciar o padrão sazonal da distribuição espacial da pluma do Amazonas no Atlântico tropical, confirmaram sua relação com as correntes de fronteira oeste. Os mesmos autores identificaram também a pluma de água Amazônica em volta da retroflexão da CNB de junho a setembro e seu deslocamento para leste pela contracorrente Norte Equatorial (CCNE). Os campos numéricos de salinidade também confirmam a existência de transporte para leste de parte desta pluma, resultante da retroflexão da $\mathrm{CNB}$, próximo de $8^{\circ} \mathrm{N}$ entre agosto e setembro (Figura 6h e 6i).

Segundo Garzoli (1992), a CCNE atinge sua máxima intensidade no verão e outono boreal quando a ZCIT se encontra no seu extremo norte, período este em que o modelo numérico registra a CNB se afastando da costa e sofrendo retroflexão para leste. No período de inverno e primavera boreal a velocidade da CCNE é mínima, associada à posição da ZCIT próximo ao Equador. Resultados do modelo registram um aumento na intensidade da retroflexão da $\mathrm{CNB}$, em torno de $50^{\circ} \mathrm{W}-8^{\circ} \mathrm{N}$, entre agosto e setembro (Figura 6h e 6i) e diminuição na intensidade de retroflexão de março a junho (Figura 6c, 6d, 6e e 6f).

Ffield (2005) discute, por meio de imagens de microondas da temperatura na superfície do mar - "TRMM Microwave Imager", a influência de água Amazônica em volta de anéis ou "rings" formados pela retroflexão da $\mathrm{CNB}$, e o deslocamento desses anéis para noroeste ao longo da costa. Este esquema, associado com a dispersão de água Amazônica em volta dos anéis foi também observado por Fratantoni e Glickson (2002) em torno de $7^{\circ} \mathrm{N}$ de julho a setembro, durante o período em que ocorre a retroflexão da $\mathrm{CNB}$. A análise mais detalhada da evolução espaço-temporal da pluma Amazônica associada a estes anéis podem ser observados nos resultados simulados pelo modelo ATL-6. Os resultados do modelo referente às médias mensais de junho a setembro registram a formação e a presença destes anéis com a influência de água Amazônica em sua volta (Figura 6f, 6g, 6h e 6i). Estes resultados também registram a diminuição da presença de água de baixa salinidade nas médias mensais dos meses de novembro a dezembro, período este em que se tem menor volume da descarga do rio Amazonas e o enfraquecimento na intensidade da CCNE (Figura 6k e 6l).

Medidas observadas in situ por Wilson et al. (2002), demonstraram que estes anéis apresentam diâmetros em volta de $370 \mathrm{~km}$. Resultado da média mensal do modelo referente a julho também registrou a presença de um anel $\left(50^{\circ} \mathrm{W}-8^{\circ}\right.$ N) com diâmetros de $340 \mathrm{~km}$ (Figura 6g). 

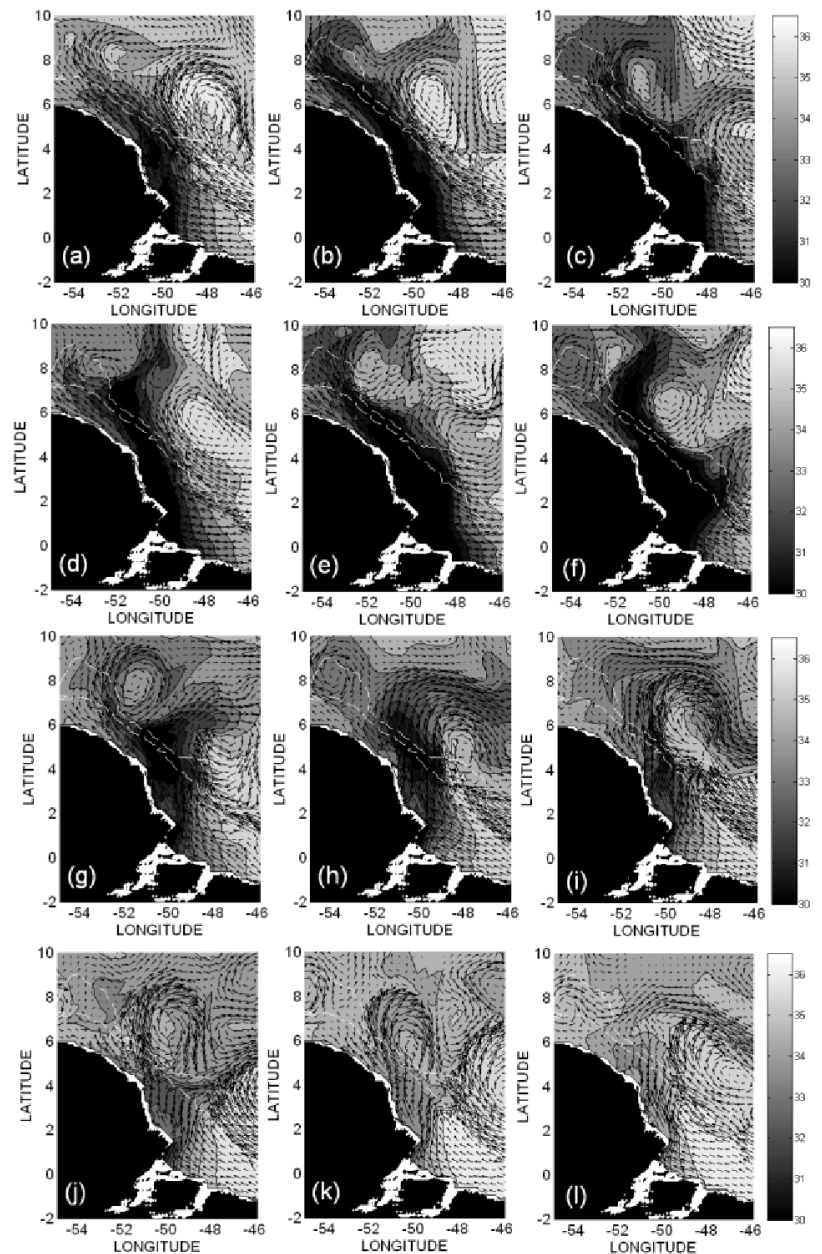

Figura 6. Análise da evolução espaço-temporal da pluma Amazônica associada às formações de anéis, simulados para as médias mensais do ano de 1999, período de campanha do Programa REVIZEE-SCORE/N, correspondentes aos meses de janeiro (a), fevereiro (b), março (c), abril (d), maio (e), junho (f), julho $(\mathrm{g})$, agosto (h), setembro (i), outubro (j), novembro (k), dezembro (l).

\section{CONCLUSÕES}

Os dados hidrográficos coletados durante o programa REVIZEE Norte integrados com os resultados do modelo ATL-6 simulados para o mesmo período, permitiram evidenciar a variabilidade sazonal e espacial da pluma Amazônica e as suas relaçôes com a dinâmica oceânica na região da costa norte brasileira. Há deslocamento sazonal da pluma na ZEE/Norte do Brasil segundo a direção dos alísios e as correntes de fronteiras. Há maior predominância da pluma de água Amazônica próximo da foz do rio Amazonas no primeiro semestre do ano, coincidente com a maior vazão do rio e dos ventos alísios de nordeste. De março a maio se tem maior deslocamento da pluma para sudeste da foz do rio Amazonas, ao longo e próximo da costa. $\mathrm{Na}$ área oceânica o deslocamento da pluma está relacionado com as correntes de fronteiras, que a deslocam para noroeste pela CNB. Em agosto e setembro, próximo de $7^{\circ} \mathrm{N}$, a pluma sofre uma retroflexão para leste alimentando a CCNE.

Resultados do modelo mostram a formação de anéis em consequiência da retroflexão da $\mathrm{CNB}$. A análise mais detalhada dos resultados simulados para as médias mensais do ano de 1999, período de campanha do Programa REVIZEE registrou a presença destes anéis com influência de água Amazônica em sua volta de agosto a setembro, tendendo a diminuir nos meses subseqüentes e desaparecendo em dezembro, associado com a variabilidade sazonal e espacial das correntes de fronteiras e vazão do rio Amazonas.

\section{AGRADECIMENTOS}

Os autores agradecem a equipe do Sub-Comitê Regional Norte do Programa REVIZEE, bem como aos comandantes e a tripulação do NOc. Antares da Marinha do Brasil, pelo esforço e dedicação demonstrados durante todas as expedições oceanográficas e a Anne Marie Treguier pela disponibilidade dos resultados do modelo ATL-6 projeto CLIPPER (Laboratoire de Physique des Océans/Brest-France).

\section{REFERÊNCIAS BIBLIOGRÁFICAS}

Arhan, M.; Tréguier, A.M.; Bourlès, B.; Michel, S. 2006. Analysis of the structure and variability of the Equatorial UnderCurrent in the Atlantic Ocean. J. Phys. Oceanogr., 36: 1502-1522.

Barnier, B.; Reynaud, T.; Beckmann, A.; Boning, C.; Molines, J-M.; Barnard, S.; Jia, Y. 2001. On the seasonal variability and eddies in the North Brazil Current: insight from model intercomparison experiments. J. Progress in Oceanogr., 44: 195-230.

Baumgartner, A.; Reichel, E. 1975. The world water balance. NewYork: Elsevier. 179 pp.

Bourlès, B.; Molinari R.L.; Johns, E.; Wilson, W.D; Leaman, K.D. 1999a. Upper layer currents in the western tropical north Atlantic (1989-1991). J. Geophys. Res., 104: 1361-1375.

Bourlès, B.; Gouriou, Y.; Chuchla, R. 1999b. On the circulation in the upper layer of the western equatorial Atlantic. J. Geophys. Res., 104: 21151-21170.

Calef, G.W; Grice, G.D. 1967. Influence of the Amazon River outflow on the ecology of the western Tropical Atlantic. II. Zooplankton abundance, copepod distribution, with remarks on the fauna of the low-salinity areas. J. Mar. Res., 25(1): 84-94.

CLIPPER Project team, 2000. 1/6 Atlantic Circulation model forced by the ECMWF climatology: preliminary results. LEGI report number CLIPPER-R2-2000, also available at www. ifremer.fr/lpo/clipper. Acesso: 16/10/08.

Comissão Interministerial Para os Recursos do Mar. 1994. IV Plano Setorial para os recursos do mar (1994-1998), Brasília-DF. $19 \mathrm{pp}$.

Evans, C.A.; Robinson, J.A.; Wilkinson, M.J.; Runco, S.; Dickerson, P.W.; Amsbury, D.L.; Lulla, K.P. 2000. The 1997-1998 El Niño: Images of floods and drought, in Dynamic Earth Environments: Remote Sensing Observations from Shuttle-Mir Missions (K. P. 
Lulla e L. V. Dessinov, eds.), John Wiley e Sons, New York, pp. 61-76, 262-263, 272-274.

Ffield, A. 2005. North Brazil Current rings viewed by TRMM Microwave Imager SST and the influence of the Amazon Plume. Deep-Sea Res. I, 52: 137-160.

Ffield, A. 2007. Amazon and Orinoco River plumes and NBC Rings: Bystanders or participants. in Hurricane events?. J. Climate, 20: 316-333.

Fratantoni, D.M.; Glickson, D.A. 2002. North Brazil Current ring generation and evolution observed with SeaWiFS. J. Phys. Oceanogr., 32: 1058-1074.

Garzoli, S.L. 1992. The Atlantic North Equatorial Countercurrent: Models and Observations. J. Geophys. Res., 97(C11): 1793117946.

Geyer, W.R.; Beardsley, R.C.; Lentz, S.J.; Candela, J.; Limeburner, R.; Johns, W.E.; Castro, B.M.; Soares, I.D. 1996. Physical oceanography of the Amazon shelf. Cont. Shelf Res., 16: 575616.

Gibbs, R.J. 1972. Water chemistry of the Amazon river. Geoch. et Cosmoch. Acta. London, 36: 1061-1066.

Grasshoff, K.; Ehrhardt, M.; Kremling, K. 1983. Methods of seawater analysis. 2ed. Verlag Chemie, Weinheim. 419pp.

Gulev, S.K., Barnier, B., Molines, J.-M., Penduff, T., and Chanut, J. 2007. Impact of spatial resolution of simulated surface water mass transformation in the Atlantic. Ocean Modelling., 19: 138-160.

Hu, C.; Montgomery, E.T.; Schmitt, R.W.; Müller-Karger, F.E. 2004. The Amazon and Orinoco River plumes in the tropical Atlantic and Caribbean Sea: Observation from space and S-PALACE floats. Deep-Sea Res., II, 1152-1171.

Johns, W.E.; Beardsley, R.C.; Candela, J.; Limeburner, R.; Castro, B.M. 1998. Annual cycle and variability of the North Brazil Current. J. Phys. Oceanogr., 28: 103-128.

Lentz, S. 1995a. Seasonal variations in the horizontal structure of the Amazon plume inferred from historical hydrographic data. J. Geophys. Res., 100(2): 2391-2400.

Lentz, S. 1995b. The Amazon river plume during AMASSEDS: Subtidal current variability and the importance of wind forcing. J. Geophys. Res., 100(2): 2377-2390.

Lentz, S.; Limeburner, R. 1995. The Amazon river plume during AMASSEDS: Spatial characteristics and salinity variability. J. Geophys. Res., 100(2): 2355-2375.

Madec, G.; Delecluse, P.; Imbard, M.; Levy, C. 1998. OPA 8.1 general circulation model reference manual. Notes de l'IPSL, Université P. et M. Curie, B102 T15-E5, 4 place Jussieu, Paris cedex 5, N 11. 91pp.

Michel, S.; Treguier, A.M. 2002. Sensitivity of the Equatorial Undercurrent to mixing parameterisations in the CLIPPER model. Rapport DRO/LPO n02-16, Oct. 2002. 35pp.

Milliman, J.D.; Boyle, E.A. 1975. Biological uptake of dissolved silica in the Amazon river estuary. Science, 189: 995-997.

Müller-Karger, F.E.; Mcclain, C.R.; Richardson, P. 1988. The dispersal of Amazon's water. Nature, 333: 56-59.
Nittrouer, C.A.; Demaster, D.J. 1986. Sedimentary processes on the Amazon continental shelf: past, present, and future research. Cont. Shelf Res., 6: 5-30.

Nittrouer, C.A.; Demaster, D.J. 1996. Oceanography of the Amazon continental shelf. Cont. Shelf Res. 16: 553-573

Oltman, R.E. 1968. Reconnaissance investigations of the discharge and water quality of the Amazon river. US Geol Surv, Circ. 552. 16pp.

Pailler, K.; Bourlès, B.; Gouriou, Y. 1999. The barrier layer in the western Atlantic Ocean. Geophys. Res. Let., 26: 2069-2072.

Reynaud, T.; Legrand, P.; Mercier, H.; Barnier, B. 1998. A new analysis of hydrographic data in the Atlantic and its application to an inverse modelling study. International WOCE Newsletter, 32: 29-31.

Richardson, P.L.; Hufford, G.; Limeburner, R.; Brown, W. 1994. North Brazil Current retroflection eddies. J. Geophys. Res., 99(3): 5081-5093.

Santos, M.L.S. 2004. Distribuição de nutrientes (Fósforo e Nitrogênio) na Plataforma Continental do Amazonas. Tese de Doutorado, Universidade Federal de Pernambuco, Recife, Pernambuco. 124pp.

Schott, F.A.; Fischer, J.; Stramma, L. 1998. Transports and pathsways of the upper-layer circulation in the western tropical Atlantic. $J$. Phys. Oceanogr., 28: 1904-1928.

Silva, A.C.; Araújo, M.; Medeiros, C.; Silva, M.; Bourlès, B. 2005a. Seasonal changes in the mixed and barrier layers in the western equatorial Atlantic. Brazilian Journal of Oceanography, 53(3/4): 83-98.

Silva, A.C.; Araújo, M.; Bourlès, B. 2005b. Variabilidade Sazonal da Estrutura de Massas de Água na Plataforma Continental do Amazonas. Revista Brasileira de Geofisica, 23(2): 145-157.

Silva, A.C.; Araújo, M.; Pinheiro, L.S. 2007a. Caracterização Hidrográfica da Plataforma Continental do Maranhão a partir de Dados Oceanográficos Medidos, Remotos e Modelados. Revista Brasileira de Geofisica, 25(3): 281-294.

Silva, A.C.; Araújo, M.; Bourlès, B. 2007b. Seasonal variability of the Amazon River plume during REVIZEE Program. Tropical Oceanography, 1: 1-22.

Smith, W.H.F.; Sandwell, D.T. 1997. Global Seafloor Topography from satellite altimetry and ship depth soundings. Science, 277: 1956-1962.

Sprintall, J.; Tomczak, M. 1992. Evidence of the barrier layer in the surface layer of the tropics. J. Geophys. Res., 97: 7305-7316.

Subramaniam, A.; Yager, P.L.; Carpenter, E.J.; Mahaffey, C.; Björkman, K.; Cooley, S.; Kustka, A.B.; Montoya, J.P.; SañudoWilhelmy, S.A.; Shipe, R.; Capone, D.G. 2008. Amazon River enhances diazotrophy and carbon sequestration in the tropical North Atlantic Ocean. Proceedings of the National Academy of Sciences, 105: 10460- 10465.

Treguier, A.M.; Gourcuff, C.; Lherminier, P.; Mercier, H.; Barnier, B.; Madec, G.; Molines, J-M.; Penduff, T.; Czeschel, L.; Böning, C. 2006. Internal and forced variability along a section between Greenland and Portugal in the CLIPPER Atlantic model. Ocean Dynamics, 56: 568-580 (DOI 10.1007/s10236-006-0069-y). 


\section{ACTA}

UNESCO, 1981. Background papers and supporting data on the Practical Salinity Scale 1978, Technical Papers Marine Sc., vol. 37, 144p.

UNESCO, 1996. Global River Discharge Data Base. Vol. I: Africa, Vol. II: Asia, and Vol. III: Europe.

Wilson, D.W.; Johns, W.E.; Garzoli, S.L. 2002. Velocity structure of North Brazil Current rings, Geophys. Res. Let., 29(8): 1273, doi:10.1029/2001GL013869.

Recebido em 20/10/2008

Aceito em 15/03/2009 
